\title{
The Path to the Future: Education of Nuclear Medicine Therapeutic Specialists as Responsible Physicians
}

\author{
Lisa Bodei ${ }^{1}$, Arturo Chiti ${ }^{2}$, Irvin M. Modlin ${ }^{3}$, Andrew M. Scott ${ }^{4}$, and Heiko Schöder ${ }^{1}$ \\ ${ }^{1}$ Molecular Imaging and Therapy Service, Department of Radiology, Memorial Sloan Kettering Cancer Center, New York, New York; \\ ${ }^{2}$ Humanitas University, Milan, Italy; ${ }^{3}$ Yale University School of Medicine, New Haven, CT; and ${ }^{4}$ Department of Molecular Imaging \\ and Therapy, Austin Health and University of Melbourne, and Tumor Targeting Laboratory, Olivia Newton-John Cancer Research \\ Institute, Melbourne, Australia
}

The best way to predict the future is to create it. -Abraham Lincoln

$\mathbf{T}$ he field of nuclear medicine now has extraordinary opportunities created by advances in imaging technologies and, in particular, the exponential increase in clinical applications of theranostic radiopharmaceuticals. Rather than being complacent, to achieve the full potential of these approaches the nuclear medicine community must redefine and redesign our field, placing it firmly at the intersection of diagnostics and therapy. We should thus emphasize the "medicine" aspect of the discipline of nuclear medicine to establish nuclear physicians as equal partners with other clinical disciplines. Ironically, this means reverting to our roots-nuclear medicine originally emerged from internal medicine and radiology to become an independent discipline. Today, nuclear medicine is either integrated into other academic departments (in the United States, usually radiology) or practiced within administratively independent departments and clinics (as in many European and global institutions). Although both structures offer advantages and disadvantages, shaping the future depends on the nuclear medicine community. This must begin with a restructuring of educational programs to train the experts needed for an expanding clinical practice and, specifically, to facilitate the rapid expansion of theranostics.

Current training programs place little emphasis on therapeutic nuclear medicine procedures. They neglect to consider that the high-quality practice of theranostics requires not only technical skills and expertise in the handling of radioactivity but also knowledge in pathology, pharmacology, and medical disease management. To own, promote, and safely practice theranostics, nuclear medicine must redesign its training curricula and develop new training pathways and fellowships.

This proposal is not unique to nuclear medicine. For example, radiation oncology emerged as a viable discipline by adopting 2 strategies. First, it differentiated itself from radiology through the therapeutic application of irradiation, and second, of considerable importance, it actively participated in the clinical management of patients with cancer from diagnosis through interdisciplinary therapy

Received Sep. 12, 2019; revision accepted Sep. 27, 2019.

For correspondence or reprints contact: Lisa Bodei, Memorial Sloan Kettering Cancer Center, 1275 York Ave., New York, CT 10065.

Email: bodeil@mskcc.org

COPYRIGHT (C) 2019 by the Society of Nuclear Medicine and Molecular Imaging. DOI: 10.2967/jnumed.119.232454 to clinical follow-up, as well as by conducting clinical trials. Essentially, being a clinician and directly engaging with patients on a routine basis is increasingly important for nuclear medicine physicians who intend to advocate for and apply modern diagnostic and therapeutic tools.

It is evident that the successful clinical introduction of new medical techniques requires a cooperative effort by physicians from different disciplines, particularly when the technique is positioned at the intersection of traditionally separate academic departments and clinical entities. For instance, the introduction of diagnostic and therapeutic endoscopy some $40 \mathrm{y}$ ago required collaboration on the part of gastroenterologists and gastrointestinal surgeons to ensure clinical competence and enable technical and scientific progress. Early on, new training curricula and dedicated fellowships were developed and new medical societies emerged. Theranostics is similarly positioned at the intersection of (at least) medical oncology and nuclear medicine. Collaboration between these disciplines with regard to clinical practice, training, and research will be integral to the clinical success of theranostics and will establish it firmly in the management of diverse malignancies. Nuclear medicine physicians now have a unique opportunity and an important responsibility to be the major contributors to this advancement.

Despite the fact that the current nuclear medicine curriculum in the United States include instruction and clinical rotations in nuclear medicine therapy and dosimetry, limitations exist (1). There is no dedicated track for more in-depth and extensive training in radionuclide therapy. Indeed, the primary emphasis of educational programs in nuclear medicine focuses on imaging and increasingly on fusion imaging with SPECT/CT, PET/CT, and PET/MRI. In comparison, in the European Union, only the major training requirements such as the length of training are defined and homogeneous. Detailed training curricula are developed at the national level and, in some countries, may vary among institutions. The Charter on Training of Medical Specialists in the European Community (2) is 7 y old and has significant shortcomings. The document simply provides a list of therapeutic applications for which a nuclear medicine specialist should receive training but fails to provide any specific requirements. A review of these training guidelines and practices indicates that nuclear medicine specialists, as currently defined, are trained largely in image interpretation and nuclear medicine physics (as it relates to imaging technologies and techniques, dosimetry, and radiation health) but lack training in the 
clinical applications of radiopharmaceuticals and clinical patient management. In addition, the current training is designed to produce nuclear medicine generalists who can both perform common procedures and interpret diverse imaging studies.

Although such training is essential in setting a foundation, it may no longer be sufficient and sustainable in the sophisticated and evolving complex medical environment of the 21 st century. Subspecialization has always occurred in nuclear medicine (e.g., nuclear cardiology), and this trend will persist in radionuclide therapy and theranostics, perhaps with an even narrower focus on a limited set of diseases at larger centers. Unless such subspecialization occurs, nuclear medicine physicians are likely to soon find themselves in a super technologist role, reporting imaging findings in descriptive terms only, rather than in a clinical context, and passively receiving orders from referring physicians for the administration of radionuclide therapies. In this scenario, nuclear medicine physicians may well be experts in their field, but their roles as full clinical partners will be diminished.

Historically in the United States, very few centers have offered radionuclide therapies that directly involve nuclear medicine physicians in patient care. In many (and perhaps in most) instances, radionuclide therapies are administered consequent to the order of a referring oncologist. By way of example, this was the model widely used for administration of Zevalin ${ }^{\circledR}$, Bexxar ${ }^{\circledR},{ }^{153} \mathrm{Sm}$-EDTMP, and ${ }^{223}$ Ra-dichloride. Similarly, in many U.S. institutions, ${ }^{131} \mathrm{I}$ therapy is administered by nuclear medicine physicians, but the entire pre- and posttherapy work-up is undertaken by a referring endocrinologist. If this model is maintained and nuclear medicine physicians accept the role of service providers without offering clinical consultations and participating in integrated patient management, we will not be accepted as clinical partners but, at best, merely as technicians, expert in the handling of radionuclides/radiopharmaceuticals, and service providers.

This abrogation of clinical responsibilities is not unique to nuclear medicine but has also eroded other areas of radiology. For instance, cardiac MR and CT may be performed in radiology departments but are regularly reported by cardiologists. In contrast, interventional radiologists have better understood that the prosperity of their discipline requires embracing clinical management, and some have rebranded themselves as interventional oncologists.

We believe there is an urgent need to restructure the training of future nuclear medicine physicians. To be on par with clinical partners in other disciplines and to actively and effectively participate in the management of patients with cancer, we need to ensure that nuclear medicine trainees are equipped with disease-specific knowledge and skill sets. In part, this may occur during the current 3-y residency training, but in some instances it may require additional fellowship training. Above all, this may entail a cultural change in our discipline whereby nuclear medicine physicians consider themselves increasingly as medical experts with a specific skill set, able to perform clinical consultations and adept at identifying and managing adverse events related to nuclear medicine therapies. In our opinion, the key to the successful practice of theranostics is direct patient management, extending from patient selection to therapy administration, monitoring and management of treatment-related side effects, and short- and midterm follow-up. This vision of our field stands in stark contrast to what some have characterized as scan readers/drug injectors functioning in dark isolated reading rooms and restricted access areas.

Paradoxically, despite the increasing availability of modern tools enabling us to underscore the meaning of the word medicine in the name of our discipline and become partners in the clinical management of patients, a lack of engagement by nuclear medicine physicians may yet result in other disciplines attempting to take ownership of these tools, despite their lack of training and knowledge required for safe administration of theranostics.

The way forward, therefore, starts with training and education. Our recommendations for nuclear medicine training programs are as follows:

- An oncology/internal medicine/endocrinology rotation should be implemented for all trainees (e.g., 3- to 1-y rotations for 4- to 5-y programs; 3- to 6-mo rotations for 3-y programs).

- Active participation in dedicated tumor boards and collaboration with clinicians in patient management should become compulsory.

- A radionuclide therapy track should be established in the curriculum and be included starting from the second year, after clinical rotation.

- A theranostics track should be implemented as dedicated fellowship.

- Nuclear medicine must be repositioned as both a diagnostic and therapeutic discipline, with dedicated sub-specialists for individual sectors.

- Nuclear medicine physicians focused on particular hematology and oncology diseases should become active participants at individual specialty meetings.

Now is the moment when we can initiate and shape the future of therapeutic nuclear medicine.

\section{DISCLOSURE}

Lisa Bodei is a consultant for AAA and Ipsen. Arturo Chiti serves on the advisory board of AAA and Blue Earth Diagnostics, has received speaker honoraria from Blue Earth Diagnostics and GE Healthcare, and has completed a PhD fellowship with Sanofi. Irvin M. Modlin is a consultant for Wren Laboratories. Andrew Scott receives research funding from Abbvie, Merck, Medimmune, Telix, ITM, and Astra Zeneca and serves as a consultant to Life Science Pharmaceuticals. Heiko Schöder was a consultant to Aileron Therapeutics until June 30, 2018 (unrelated to the current work). No other potential conflict of interest relevant to this article was reported.

\section{REFERENCES}

1. Segall GM. The distinction between an AU and an ABNM-certified MD. J Nucl Med. 2019;60(7):10N.

2. Prigent A, Huic D, Costa DC. Syllabus for postgraduate specialization in nuclear medicine: 2011/2012 update-nuclear medicine training in the European Union. Eur J Nucl Med Mol Imaging. 2012;39:739-743. 